\title{
Combining Ability and Heterosis Studies in Bitter Guard (Momordica charactia L.)
}

\author{
Vibha Mishra* and D.K. Singh \\ Department of Vegetable Science, GBPUAT, Pantnagar (UK) \\ *Corresponding author
}

\begin{tabular}{|c|c|}
\hline \multicolumn{2}{|r|}{ A B S T R A C T } \\
\hline & \multirow{5}{*}{ 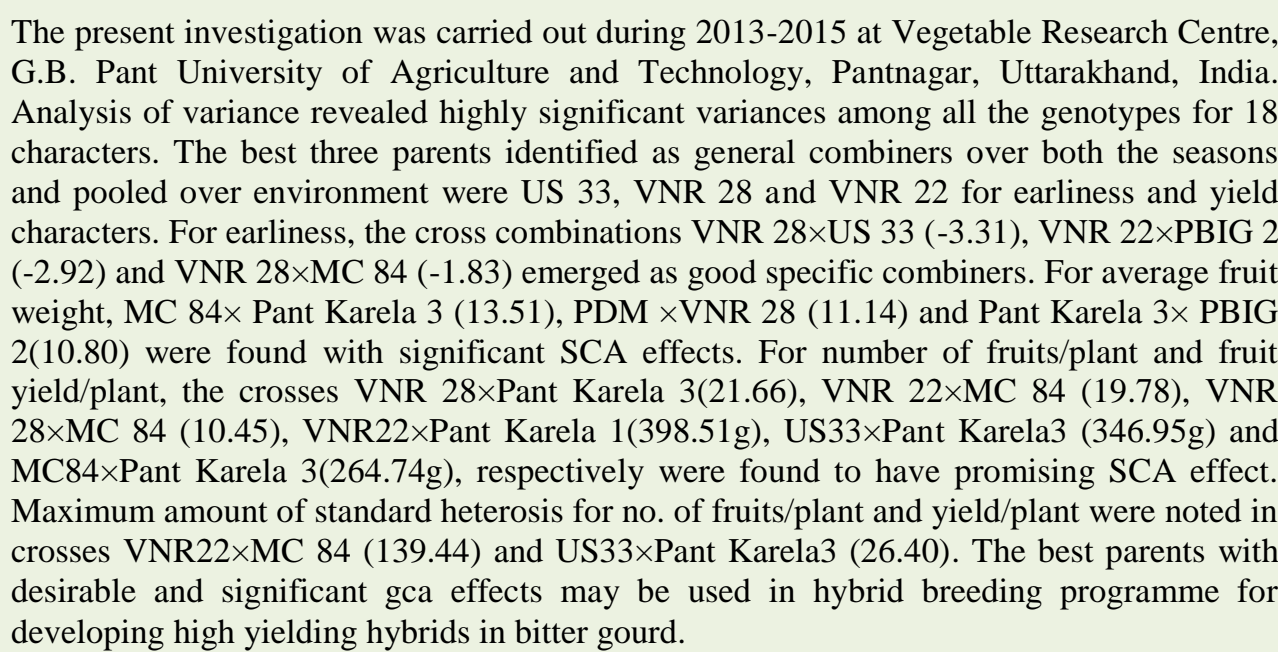 } \\
\hline & \\
\hline Article Info & \\
\hline & \\
\hline & \\
\hline
\end{tabular}

\section{Introduction}

Bitter Gourd (Momordica charantia L., $2 n=2 x=22$ ) is a multipurpose herb belonging to family Cucurbitaceous. The crop is extensively grown in India, China, Japan, South East Asia, tropical Africa and South America. Asian M. charantia originated from tropical Africa (Schaefer and Renner, 2010), while its original place of domestication is unknown yet. Areas of Eastern India and Southern China have been proposed as places of origin (Dey et al., 2006). Among the cultivated cucurbits, bitter gourd has been identified as one of the potent vegetables for export by Agricultural Processed Food Products and Export Development Authority. In India, Uttar Pradesh, Bihar, West Bengal, Orissa, Karnataka, Maharashtra, Telangana, Tamil Nadu, Kerala and Chhattisgarh are the major bitter gourd growing states with Telangana being the leading producer followed by Chhattisgarh and Orissa One of the possible approaches for achieving the targeted production is to identify and develop suitable hybrids with high yield and good 
quality. In spite of wide range of diversity very little work has been undertaken to exploit this naturally endowed diversity in the form of hybrid breeding. Hybrids in most of the vegetable crops offer opportunity of earliness, high yield, quality improvement besides better capacity to face biotic and abiotic stresses. The exploitation of heterosis is much easier in cross pollinated crops and bitter gourd being monoecious, provides ample scope for utilization of hybrid vigour on commercial scale. A wide range of variability in vegetative and fruit characters is available in bitter gourd so, the diversified parents from different locations with high yield and quality would also pave way for the development and release of hybrids having high yield, earliness and quality through heterosis breeding. Information on combining ability facilitates the choice of suitable parents for hybridization programme to develop promising $F_{1}$ hybrids. In actual plant breeding combining abilities have found their principle use in predicting the performance of parents and hybrid population. Diallel analysis is widely used to estimate combining ability effects of the parents and the crosses (Griffin, 1956). It is the most balanced and systematic experimental design to examine continuous variation. The genetic information related to parental population become available quite in early generation i.e. in $\mathrm{F}_{1}$ and it is thus useful to define breeding strategy without lossing much time. Diallel analysis provides reliable information on the components of variance, general combining ability (GCA), specific combining ability (SCA), variances and their effects (Singh and Narayanan, 1993) and also helps in formulating the breeding methodology for crop improvement.

The information usually needed for developing high yielding crop in particular species pertains to the extent of genetic variability for desirable traits in the available germplasm. Large variability ensures better chances to produce new forms. Though bitter gourd is an important cucurbitaceous vegetable and lot of variation is present for characters, such information is inadequate. Keeping in view all the above standpoint in consideration, the present investigation was conducted to study the magnitude of heterosis and combining ability of parental lines and crosses.

\section{Materials and Methods}

The present investigation was carried out at the Vegetable Research Centre, G.B.Pant University of Agriculture and Technology, Pantnagar, U.S.Nagar, during spring-summer seasons of 2013-15. Pantnagar lies on $29^{\circ}$ North latitude, $79.3^{\circ}$ East longitude and at an altitude of 243.83 meters above mean sea level and comes under the Tarai belt of Shivalik ranges of Himalayas. The climate of Pantnagar is broadly humid and subtropical in nature with hot summers and cool winters. The soil of experimental field was calcareous and of miscellaneous type and it is generally 1.0 to 1.5 meter deep with good drainage and nearly neutral reaction ( $\mathrm{pH}$ 6.0-7.5). High rainfall is generally received from June to September. The experimental material consisted of eight inbred lines of bitter gourd viz. PDM, VNR-28, VNR-22, MC-84, Pant Karela 1, US-33, Pant karela 3 and PBIG-2. Their $28 \mathrm{~F}_{1}$ 's developed by crossing in diallel fashion excluding reciprocals. The seeds of parental lines were obtained from cucurbits breeding programme of the department of Vegetable Science, G.B.Pant University of Agriculture and Technology, Pantnagar. The experiment was laid out in Randomized Block Design (RBD) with three replications. Each genotypes consisted of 10 plants. Row to row spacing was kept $3 \mathrm{~m}$, while plant to plant was kept $80 \mathrm{~cm}$, respectively. Initially, 3-4 seeds were sown per hill from which 2 plants were retained after thinning. The observations were recorded on five randomly selected plants and the average was computed for the following 24 morphological characters among first male 
flower anthesis (days), first female flower anthesis (days), node number to first male flower, node number to first female flower, number of fruits per plant, average fruit weight $(\mathrm{g})$, fruit length $(\mathrm{cm})$, fruit diameter $(\mathrm{cm}), \mathrm{L} / \mathrm{D}$ ratio, main vine length $(\mathrm{m})$, number of primary branches per vine, internodal length $(\mathrm{cm})$, leaf blade length $(\mathrm{cm})$, leaf blade width $(\mathrm{cm})$, petiole length $(\mathrm{cm})$, leaf area $\left(\mathrm{cm}^{2}\right)$, fruit yield /ha (q).

\section{Statistical analysis}

The data was statistically analyzed following the standard procedure as applicable to a typical randomized block design. Treatments were tested by 'F-test' (Snedecor and Cochran, 1967). Heterosis expressed as per cent increase or decrease in the performance of $F_{1}$ over mid-parent (average or relative) heterosis, better parent (heterobeltiosis) and check parent (standard heterosis).

Residual heterosis was calculated using similar formulas instead of $F_{1}$ mean was used for all the genotypes under study. The combining ability analysis for parental genotypes and their crosses were carried out following method 2 and Model I of Griffing (1956).

\section{Results and Discussion}

\section{Combining ability analysis}

\section{Analysis of variance for combining ability}

The analysis of variances of combining ability was done for the eighteen characters in bitter gourd (Table 1). The GCA variances were highly significant for all the characters for both the season and pooled over season except internodal length in pooled season. The SCA variances were also highly significant for all the characters. The GCA variances were higher and prominent than SCA variances for all the characters under study.

\section{Estimates of general combining ability effects}

The estimates of general combining ability (GCA) of the parents for various characters for both the season and pooled have been shown in Table $2 \mathrm{a}$ and $2 \mathrm{~b}$. For days to first male and female flower and node no. to first male and female flower, the negative gca and sca effects were considered to be desirable as it indicates earliness. The parent VNR-28 was recorded as the best general combiner for the traits first male flower anthesis, first female flower anthesis, node no. to first male flower and node no. to first female flower and US-33 for petiole length, leaf area, fruit length, fruit diameter, L/D ratio, average fruit weight, fruit yield per plant and fruit yield per ha. Whereas, the parent VNR-22 was found to be the best general combiners for main vine length and internodal length. These lines may be used in bitter gourd improvement programme for developing desirable genotypes. GCA effects would be more stable as compared to SCA effects. In general, additive effects are mainly due to polygenes producing fixable effects and indicate the capacity of variety in relation to all other varieties, it was crossed with. High GCA effects of a parent is a function of breeding value and hence due to additive gene effect and/or additive $\times$ additive interaction effect which represents the fixable components of genetic variance (Griffing, 1956). Apparently, parents with good GCA effects may be presumed to possess more favourable genes for the concerned traits. The findings were in proximity to those of the studies conducted by Srivastava and Nath (1983) and Bhatt et al., (2017) who observed significantly high GCA and SCA effects were for days to flowering, fruit per plant, fruit weight and total yield per plant in majority of parents. Gopalkrishnan (1986) also evaluated 30 crosses and reported parent MDU-1 as best general combiner for weight, size, number of fruits per plant and total yield and the cross, 
Priya $\times$ MDU-1 was reported to have high SCA effect. Vahab (1989) reported Priya, MC-66, and MC-84 as best general combiner for total yield. Devadas et al., (1993) also reported $c v$. MC 13 as good general combiner for seeds per fruit and 100 seed weight and MC 84 for field emergence, seedling length and seedling dry weight. Gupta et al., (2006) reported highly significant general combining ability (GCA) and specific combining ability (SCA) for yield and yield components indicating the presence of variability in combining ability of the parents. The similar sort of studies were also conducted by Tamilselvi et al., (2015) idenitifed the parents Kasi Harit, Vadhalagundu Local and CO2 as the best genotypes for improvement of earliness and yield characters

\section{Specific combining ability studies}

Specific combining ability effect which represents the predominance of non additive gene action is a major component that may be utilized in heterosis breeding (Table 3 ). Out of 28, cross combinations 3,11 and 17 exhibited significant and desirable sca effects for days to first male anthesis during 2014, 2015 and pooled over analysis. The cross combination VNR-22 x US-33 had exhibited the highest significant sca effects for first male flower anthesis over all the season and pooled over analysis and VNR-22 x MC-84. For node no. of first female flower, the estimate of sca effect of crosses in first season were found significant negative effects in VNR 28× US 33 (-7.25), MC $84 \times$ US $33(-3.82)$ and VNR 28 $\times$ VNR 22 (-3.24). Similarly, for season II, the crosses revealed significant effects were VNR $28 \times$ US 33 (-5.46), MC $84 \times$ US 33 (-3.22) and MC $84 \times$ PBIG 2 (-3.16). For internodal length for the year 2014 and 2015, significant sca effects were observed in almost all the crosses. The highest sca effect was observed for the crosses Pant karela 1× Pant karela 3 (1.03 and 1.09), followed by VNR $28 \times$ VNR 22 (0.95 and 0.98) and VNR 22× US33 (0.74 and 1.14) in season I and II. For pooled season also similar crosses showed highest significant sca effects. Among 28 crosses, maximum positively associated values for sca effects for fruit length were recorded in cross combinations MC $84 \times$ PBIG 2 (4.82), VNR22 $\times$ US 33 (4.35) and PDM $\times$ VNR 28 (2.36) for season I and in cross combinations MC 84×PBIG 2 (5.53), VNR22×US 33 (3.25) and VNR $28 \times$ US 33 (3.25) for season II. For pooled season, crosses with maximum sca effect were MC 84× PBIG 2 (5.17), VNR 22× US 33 (3.71) and PDM $\times$ Pant karela 1 (2.39).

For fruit weight, estimates of sca effects revealed that Pant karela $3 \times$ PBIG 2 (11.59 and 10.01), MC 84× Pant karela 3 (15.98 and 11.03) showed maximum value of sca for both the season, while PDM $\times$ VNR 28 (12.52) in season I and VNR 28× US 33 (10.61) in season II. Pooled data showed similar crosses with highest values viz., Pant karela $3 \times$ PBIG 2 (10.80), MC 84× Pant karela 3 (13.51) and PDM $\times$ VNR 28 (11.14). Whereas for number of fruits/plant, the perusal of results revealed highest value of $31.74,20.27$ and 19.54 for VNR 28×Pant karela 3, VNR 28× MC 84 and VNR 22×MC 84, respectively in 2014 season. VNR $28 \times$ Pant karela 3, VNR $22 \times$ MC 84 and PDM $\times$ Pant karela 1 (7.09) noted maximum value for season II. Similarly, VNR $28 \times$ Pant karela 3 (21.66), VNR 28× MC 84 (10.45) and VNR 22×MC 84 (19.78) showed significant highest sca effects. Fruit yield/ha showed maximum values of significant sca effects in crosses US 33× Pant karela 3 (14.46 and 14.48), VNR 22× Pant karela 1 (17.26 and 16.88) for both seasons. Whereas, US $33 \times$ PBIG 2 (13.32) in 2014 and MC 84xPant karela 3 (10.44) in 2015 having highest sca effect. Pooled season showed US 33× Pant karela 3 (14.47), VNR 22× Pant karela 1 (17.07) and MC $84 \times$ Pant karela 3 (11.05) with maximum values. 
Table.1 ANOVA for combining ability for various quantitative traits

\begin{tabular}{|c|c|c|c|c|c|c|c|c|c|c|c|c|}
\hline \multirow[t]{2}{*}{ Characters } & \multicolumn{3}{|c|}{2014} & \multicolumn{3}{|c|}{2015} & \multicolumn{6}{|c|}{ Pooled } \\
\hline & GCA & SCA & Error & GCA & SCA & Error & GCA & SCA & Env. & GCA*Env. & SCA*Env & Error \\
\hline d.f. & 7 & 28 & 70 & 7 & 28 & 70 & 7 & 28 & 1 & 7 & 28 & 140 \\
\hline $\begin{array}{l}\text { 1st Male Flower Anthesis } \\
\text { (days) }\end{array}$ & $45.72 * *$ & $1.18^{* *}$ & 0.36 & $30.51 * *$ & $14.89 * *$ & 0.35 & $49.53 * *$ & $9.40 * *$ & $74.16^{* *}$ & $26.70 * *$ & $6.66^{* *}$ & 0.36 \\
\hline $\begin{array}{l}\text { 1st Female Flower Anthesis } \\
\text { (days) }\end{array}$ & $40.05 * *$ & $4.90 * *$ & 0.42 & $63.63 * *$ & $8.32 * *$ & 0.43 & $85.34 * *$ & $5.61 * *$ & $233.00 * *$ & $18.34 * *$ & $7.61 * *$ & 0.43 \\
\hline Node No. of 1st Male Flower & $27.18 * *$ & $1.73^{* *}$ & 0.03 & $31.23 * *$ & $31.42 * *$ & 0.03 & $47.58 * *$ & $16.03 * *$ & $24.81 * *$ & $10.83 * *$ & $17.11 * *$ & 0.03 \\
\hline Node No. of 1st Female Flower & $98.39 * *$ & $7.08 * *$ & 0.12 & $69.04 * *$ & $8.94 * *$ & 0.1 & $160.81 * *$ & $12.33 * *$ & $5.49 * *$ & $6.62 * *$ & $3.69 * *$ & 0.11 \\
\hline Main Vine Length (cm) & $1.06 * *$ & $0.58 * *$ & 0 & $0.41 * *$ & $0.25 * *$ & 0 & $0.85 * *$ & $0.44 * *$ & $2.85^{* *}$ & $0.62 * *$ & $0.39 * *$ & 0 \\
\hline Primary Branches/ Plant & $2.56^{* *}$ & $14.44 * *$ & 0.05 & $12.65 * *$ & $4.16^{* *}$ & 0.05 & $9.03 * *$ & $9.69 * *$ & $196.01 * *$ & $6.18^{* *}$ & $8.92 * *$ & 0.05 \\
\hline Internodal Length (cm) & $0.49 * *$ & $0.48 * *$ & 0.01 & $0.44 * *$ & $0.60 * *$ & 0.01 & $0.92 * *$ & $1.06 * *$ & $1.20 * *$ & 0 & $0.02 * *$ & 0.01 \\
\hline Leaf Length (cm) & $8.28 * *$ & $7.53 * *$ & 0.02 & $9.96 * *$ & $4.12 * *$ & 0.02 & $17.15^{* *}$ & $10.57 * *$ & $7.12 * *$ & $1.10 * *$ & $1.08 * *$ & 0.02 \\
\hline Leaf Width (cm) & $2.52 * *$ & $2.28 * *$ & 0.02 & $3.72 * *$ & $2.17 * *$ & 0.02 & $5.87 * *$ & $4.04 * *$ & $0.80 * *$ & $0.36 * *$ & $0.41 * *$ & 0.02 \\
\hline Petiole Length (cm) & $1.59 * *$ & $2.86^{* *}$ & 0 & $5.23 * *$ & $3.15^{* *}$ & 0.01 & $4.24 * *$ & $4.39 * *$ & $33.70^{* *}$ & $2.59 * *$ & $1.62 * *$ & 0.01 \\
\hline Leaf Area $\left(\mathrm{cm}^{2}\right)$ & $1620.78 * *$ & $1412.75^{* *}$ & 3.29 & $1710.50 * *$ & $906.97 * *$ & 2.86 & $3144.35 * *$ & $2150.55 * *$ & $951.81 * *$ & $186.93 * *$ & $169.17 * *$ & 3.07 \\
\hline Fruit Length (cm) & $45.62 * *$ & $6.43 * *$ & 0.05 & $38.72 * *$ & $5.66^{* *}$ & 0.05 & $81.85 * *$ & $10.93 * *$ & $9.96 * *$ & $2.49 * *$ & $1.16^{* *}$ & 0.05 \\
\hline Fruit diameter (cm) & $0.48^{* *}$ & $0.09 * *$ & 0 & $0.30 * *$ & $0.09 * *$ & 0 & $0.69 * *$ & $0.13 * *$ & $0.88 * *$ & $0.10 * *$ & $0.05 * *$ & 0 \\
\hline Length/ diameter ratio & $3.59 * *$ & $0.43 * *$ & 0.01 & $3.02 * *$ & $0.36 * *$ & 0.01 & $6.50 * *$ & $0.70 * *$ & $0.04 *$ & $0.12 * *$ & $0.09 * *$ & 0.01 \\
\hline Average Fruit Weight (g) & $510.84 * *$ & $128.72 * *$ & 0.66 & $538.21^{* *}$ & $121.89 * *$ & 0.57 & $1015.59 * *$ & $233.34 * *$ & $223.29 * *$ & $33.45 * *$ & $17.28 * *$ & 0.62 \\
\hline Fruits/ Plant & $407.09 * *$ & $131.41 * *$ & 0.28 & $241.40 * *$ & $61.03 * *$ & 0.27 & $621.28 * *$ & $161.17 * *$ & $369.59 * *$ & $27.21 * *$ & $31.27 * *$ & 0.27 \\
\hline Fruit Yield//Plant (g) & $\begin{array}{c}82731.06^{*} \\
*\end{array}$ & $\begin{array}{c}65283.28 * \\
*\end{array}$ & 442.82 & $\begin{array}{l}83106.71 * \\
*\end{array}$ & $\begin{array}{c}51911.91 * \\
*\end{array}$ & 656.73 & $\begin{array}{l}153485.25^{*} \\
*\end{array}$ & $\begin{array}{l}108280.39 * \\
*\end{array}$ & $\begin{array}{l}33139.22 * \\
*\end{array}$ & $\begin{array}{c}12352.51 * \\
*\end{array}$ & $\begin{array}{l}8914.80 * \\
*\end{array}$ & $\begin{array}{c}549.7 \\
8\end{array}$ \\
\hline Fruit Yield/ hac & $143.65 * *$ & $113.36^{* *}$ & 1.11 & $142.82 * *$ & $91.46^{* *}$ & 1.05 & $265.01 * *$ & $189.37 * *$ & $55.21 * *$ & $21.46^{* *}$ & $15.45 * *$ & 1.08 \\
\hline
\end{tabular}


Table.2a Estimates of GCA traits over pooled analysis

\begin{tabular}{|c|c|c|c|c|c|c|c|c|c|}
\hline $\begin{array}{l}\text { Source of } \\
\text { variation }\end{array}$ & $\begin{array}{c}\text { First male } \\
\text { flower } \\
\text { anthesis } \\
\text { (days) }\end{array}$ & $\begin{array}{c}\text { First female } \\
\text { flower } \\
\text { anthesis } \\
\text { (days) }\end{array}$ & $\begin{array}{l}\text { Node no. to } \\
\text { first male } \\
\text { flower }\end{array}$ & $\begin{array}{l}\text { Node no. to } \\
\text { first female } \\
\text { flower }\end{array}$ & $\begin{array}{l}\text { Main vine } \\
\text { length }(\mathrm{m})\end{array}$ & $\begin{array}{c}\text { No.of } \\
\text { primary } \\
\text { branch }\end{array}$ & $\begin{array}{l}\text { Inter-nodal } \\
\text { length }(\mathrm{cm})\end{array}$ & $\begin{array}{c}\text { Leaf } \\
\operatorname{length}(\mathbf{c m})\end{array}$ & $\begin{array}{c}\text { Leaf width } \\
\text { (cm) }\end{array}$ \\
\hline PDM & -0.23 & $-2.06 * *$ & $2.71 * *$ & $0.77 * *$ & $0.03 * *$ & $-0.51 * *$ & $-0.20 * *$ & $-0.11 * *$ & $-0.25 * *$ \\
\hline VNR-28 & $-2.32 * *$ & $-3.46 * *$ & $-2.55 * *$ & $-4.74 * *$ & $-0.26 * *$ & $-0.34 * *$ & $-0.20 * *$ & $0.75 * *$ & $0.18 * *$ \\
\hline VNR-22 & $0.55^{* *}$ & $0.43 * *$ & $0.85^{* *}$ & $0.71 * *$ & $0.28 * *$ & $-0.72 * *$ & $0.46^{* *}$ & $-0.68 * *$ & 0.05 \\
\hline MC-84 & $0.48 * *$ & 0.17 & $-0.63 * *$ & $-2.50 * *$ & $0.19 * *$ & $-0.61 * *$ & $-0.08 * *$ & $-1.29 * *$ & $-0.70 * *$ \\
\hline PK-1 & $-1.65 * *$ & $-0.64 * *$ & 0.05 & $0.58 * *$ & $-0.17 * *$ & $0.15^{* *}$ & $0.07 * *$ & $0.60 * *$ & $0.49 * *$ \\
\hline US-33 & $2.93 * *$ & $2.74 * *$ & $0.81 * *$ & $4.77 * *$ & $-0.14 * *$ & $0.35^{* *}$ & 0.02 & $1.00 * *$ & $0.81 * *$ \\
\hline PK-3 & 0.02 & $2.28 * *$ & $-0.97 * *$ & $1.48 * *$ & $0.22 * *$ & $1.24 * *$ & $0.05^{* *}$ & $-1.11^{* *}$ & $-0.74 * *$ \\
\hline PBIG-2 & 0.22 & $0.54 * *$ & $-0.27 * *$ & $-1.08 * *$ & $-0.15^{* *}$ & $0.46^{* *}$ & $-0.11 * *$ & $0.84 * *$ & $0.16^{* *}$ \\
\hline \multicolumn{10}{|l|}{ CD for GCA } \\
\hline Gi--Gj at $95 \%$ & 0.37 & 0.41 & 0.11 & 0.21 & 0.03 & 0.14 & 0.05 & 0.09 & 0.08 \\
\hline Gi--Gj at $99 \%$ & 0.49 & 0.54 & 0.15 & 0.27 & 0.04 & 0.18 & 0.07 & 0.12 & 0.11 \\
\hline
\end{tabular}


Table.2b Estimates of GCA traits over pooled analysis

\begin{tabular}{|c|c|c|c|c|c|c|c|c|c|}
\hline $\begin{array}{l}\text { Source of } \\
\text { variation }\end{array}$ & $\begin{array}{c}\text { Petiole } \\
\text { length } \\
\text { (cm) }\end{array}$ & $\begin{array}{c}\text { Leaf area } \\
\left(\mathrm{cm}^{2}\right)\end{array}$ & $\begin{array}{c}\text { Fruit } \\
\text { length } \\
\text { (cm) }\end{array}$ & $\begin{array}{l}\text { Fruit dia. } \\
\quad(\mathrm{cm})\end{array}$ & L/D ratio & $\begin{array}{l}\text { Aver-age } \\
\text { fruit } \\
\text { weight (g) }\end{array}$ & $\begin{array}{l}\text { No. of } \\
\text { fruits } \\
\text { /plant }\end{array}$ & $\begin{array}{c}\text { Fruit } \\
\text { yield } \\
\text { /plant (g) }\end{array}$ & $\begin{array}{c}\text { Fruit } \\
\text { yield/ha } \\
\text { (q/ ha) }\end{array}$ \\
\hline PDM & $-0.49 * *$ & $-4.73 * *$ & $1.21 * *$ & 0 & $0.26 * *$ & $7.66^{* *}$ & $-6.34 * *$ & $78.75^{* *}$ & $3.27 * *$ \\
\hline VNR-28 & $0.18 * *$ & $5.81 * *$ & $-4.27 * *$ & $0.14 * *$ & $-1.09 * *$ & $-11.17 * *$ & $11.55^{* *}$ & 9.38 & 0.38 \\
\hline VNR-22 & $-0.16 * *$ & $-6.58 * *$ & $2.06 * *$ & $-0.29 * *$ & $0.78 * *$ & $-7.05^{* *}$ & $3.01 * *$ & $-58.82 * *$ & $-2.41 * *$ \\
\hline MC-84 & $-0.45^{* *}$ & $-15.09 * *$ & $-0.41 * *$ & $0.12 * *$ & $-0.22 * *$ & $0.49 * *$ & $1.68 * *$ & $57.07 * *$ & $2.36^{* *}$ \\
\hline PK-1 & $0.20 * *$ & $7.96 * *$ & -0.09 & $-0.27 * *$ & $0.21 * *$ & $0.42 *$ & $-1.82 * *$ & $-53.43 * *$ & $-2.18^{* *}$ \\
\hline US-33 & $0.83 * *$ & $20.44 * *$ & $2.09 * *$ & $0.16^{* *}$ & $0.48 * *$ & $10.79 * *$ & $-4.43 * *$ & $128.50 * *$ & $5.34 * *$ \\
\hline PK-3 & $-0.41 * *$ & $-15.34 * *$ & $-0.23^{* *}$ & 0 & $-0.19 * *$ & $0.99 * *$ & $-0.77 * *$ & $-16.24 * *$ & $-0.69 * *$ \\
\hline PBIG-2 & $0.30 * *$ & $7.54 * *$ & $-0.37 * *$ & $0.15^{* *}$ & $-0.22 * *$ & $-2.13 * *$ & $-2.88 * *$ & $-145.20 * *$ & $-6.06 * *$ \\
\hline \multicolumn{10}{|l|}{$\begin{array}{l}\text { CD for } \\
\text { GCA }\end{array}$} \\
\hline $\begin{array}{l}\text { Gi--Gj at } \\
95 \%\end{array}$ & 0.05 & 1.1 & 0.14 & 0.04 & 0.06 & 0.49 & 0.33 & 14.66 & 0.65 \\
\hline $\begin{array}{l}\text { Gi--Gj at } \\
99 \%\end{array}$ & 0.06 & 1.45 & 0.19 & 0.05 & 0.07 & 0.65 & 0.43 & 19.36 & 0.86 \\
\hline
\end{tabular}


Table.3 Three best hybrids in terms of specific combining ability, standard heterosis and heterobeltiosis

\begin{tabular}{|c|c|c|c|c|}
\hline S. No. & Characters & Best Specific Combiners & Best Crosses (Standard Heterosis) & Best Crosses (Heterobeltiosis) \\
\hline 1. & $\begin{array}{l}\text { 1st Male Flower Anthesis } \\
\text { (days) }\end{array}$ & $\begin{array}{l}\text { VNR } 22 \times \text { US } 33(-4.35), \text { VNR } 28 \times \text { PBIG } 4(- \\
3.39), \text { VNR } 22 \times \text { MC } 84(-2.43)\end{array}$ & $\begin{array}{l}\text { VNR28×Pant Karela3(-18.55), PDM×VNR28(- } \\
\text { 14.85), VNR28× MC84 (-13.00) }\end{array}$ & $\begin{array}{l}\text { VNR28×US33 (-20.91), VNR22×US33 (-19.70), } \\
\text { VNR28× Pant Karela 3(-18.55) }\end{array}$ \\
\hline 2. & $\begin{array}{l}\text { 1st Female Flower Anthesis } \\
\text { (days) }\end{array}$ & $\begin{array}{l}\text { VNR } 28 \times \text { US } 33(-3.31), \text { VNR } 22 \times \text { PBIG } 2(- \\
2.92) \text {, VNR } 28 \times \text { MC } 84(-1.83)\end{array}$ & $\begin{array}{l}\text { PDM } \times \text { VNR28 (-23.95), VNR } 28 \times \text { MC } 84(-21.95) \\
\text { VNR } 28 \times \text { US33 }(-19.77)\end{array}$ & $\begin{array}{l}\text { VNR } 28 \times \text { US33 (-20.00), VNR28× Pant Karela 3(- } \\
\text { 15.60), VNR } 28 \times \text { MC84 (-13.19) }\end{array}$ \\
\hline 3. & $\begin{array}{l}\text { Node No. of 1st Male } \\
\text { Flower }\end{array}$ & $\begin{array}{l}\text { PBIG3×PBIG4(-2.42) } \\
\text { PDM } \times \text { PBIG } 3(-1.46)\end{array}$ & $\begin{array}{l}\text { VNR28×PBIG2 (-15.24), VNR28 } \times \\
\text { PantKarela3(13.83), Pant Karela1×Pant Karela 3(- } \\
\text { 7.40) }\end{array}$ & $\begin{array}{l}\text { VNR28× US33 (-30.83), Pant Karela 1× US33(- } \\
\text { 27.76), VNR 28×VNR } 22(-26.88)\end{array}$ \\
\hline 4. & $\begin{array}{l}\text { Node No. of 1st Female } \\
\text { Flower, }\end{array}$ & $\begin{array}{l}\text { VNR } 28 \times \text { US } 33(-6.35), \text { MC } 84 \times \text { US } 33(- \\
3.52) \text {, MC } 84 \times \text { PBIG } 2(-2.04)\end{array}$ & $\begin{array}{l}\text { VNR 28× MC } 84 \text { (-36.72), VNR 28× US } 33(- \\
\text { 36.51), VNR 28×PBIG } 2(-29.44)\end{array}$ & $\begin{array}{l}\text { VNR28×US33 (-55.87), MC84×US 33(-40.13), } \\
\text { VNR } 28 \times \text { VNR 22(- 26.08) }\end{array}$ \\
\hline 5. & Main Vine Length $(\mathrm{cm})$ & $\begin{array}{l}\text { MC } 84 \times \text { PBIG } 4(0.70), \text { MC } 84 \times \text { US } 33(0.68) \text {, } \\
\text { PDM } \times \text { PBIG } 2(0.60)\end{array}$ & $\begin{array}{l}\text { MC } 84 \times \text { Pant Karela 3(49.54), VNR 22× Pant } \\
\text { Karela } 3 \text { (44.14), MC } 84 \times \text { US } 33 \text { (36.03) }\end{array}$ & $\begin{array}{l}\text { US33×Pant Karela } 3 \text { (33.33), VNR } 22 \times \text { Pant Karela } \\
3 \text { (21.22), Pant Karela } 1 \times \text { Pant Karela } 3(17.10)\end{array}$ \\
\hline 6. & Primary Branches/ Plant & $\begin{array}{l}\text { MC } 84 \times \text { US 33(5.15), PBIG } 4 \times \text { PBIG2 (3.59), } \\
\text { PDM } \times \text { PBIG } 2(3.44)\end{array}$ & $\begin{array}{l}\text { Pant Karela } 3 \times \text { PBIG 2(40.00), MC } 84 \times \text { US } \\
\text { 33(36.92), US 33×Pant Karela } 3(33.84)\end{array}$ & $\begin{array}{l}\text { MC 84×US 33(50.85), PDM× PBIG 2(40.52), Pant } \\
\text { Karela 3× PBIG 2(40.00) }\end{array}$ \\
\hline 7. & Internodal Length $(\mathrm{cm})$ & $\begin{array}{l}\text { Pant K } 1 \times \text { Pant K } 3(1.09), \text { VNR } 28 \times \text { VNR } \\
22(0.98), \text { VNR } 22 \times \text { US33 (1.14) }\end{array}$ & $\begin{array}{l}\text { VNR } 22 \times \text { US } 33 \text { (30.43), Pant Karela } 1 \times \text { Pant } \\
\text { Karela } 3(26.95) \text {, VNR } 22 \times \text { Pant Karela } 3(23.48)\end{array}$ & $\begin{array}{l}\text { VNR } 28 \times \text { VNR } 22 \text { (30.36), VNR } 22 \times \text { Pant Karela } 3 \\
(23.48), \text { VNR } 22 \times \text { US } 33(16.28)\end{array}$ \\
\hline 8. & Leaf Length $(\mathrm{cm})$ & $\begin{array}{l}\text { PDM } \times \text { VNR22(3.00), VNR } 22 \times \text { MC } 84(3.10), \\
\text { VNR } 28 \times \text { MC } 84(2.91)\end{array}$ & $\begin{array}{l}\text { VNR28×Pantkarela } 1(127.38), \text { VNR28× MC84 } \\
(112.29), \text { PDM } \times \text { VNR22 (109.75) }\end{array}$ & $\begin{array}{l}\text { VNR 22×MC } 84 \text { (39.69), MC } 84 \times \text { Pant Karela } 3 \\
(30.98), \text { VNR } 28 \times \text { MC } 84 \text { (19.41) }\end{array}$ \\
\hline 9. & Leaf Width $(\mathrm{cm})$ & $\begin{array}{l}\text { VNR22×MC84(2.12) VNR28×MC } 84(1.83), \\
\text { PDM } \times \text { PantKarela 1(1.70) }\end{array}$ & $\begin{array}{l}\text { PDM } \times \text { Pant Karela } 1(117.90), \text { VNR } 28 \times \text { MC } 84 \\
(103.26), \text { VNR } 28 \times \text { Pant Karela } 1(101.98)\end{array}$ & $\begin{array}{l}\text { MC 84×Pant Karela3 (38.28), VNR 22×MC84 } \\
(29.63), \text { PDM } \times \text { VNR } 22(23.12)\end{array}$ \\
\hline 10. & Petiole Length $(\mathrm{cm})$ & $\begin{array}{l}\text { PDM } \times \text { VNR 22(2.25),, VNR 28×MC } \\
84(2.76) \text { PDM } \times \text { Pant Karela 3(1.36) }\end{array}$ & - & $\begin{array}{l}\text { MC } 84 \times \text { Pant Karela } 3 \text { (69.88), } \\
\text { VNR28×MC84(68.73), VNR22×MC } 84 \text { (50.58), }\end{array}$ \\
\hline 11. & Leaf Area $\left(\mathrm{cm}^{2}\right)$ & $\begin{array}{l}\text { VNR } 22 \times \text { MC } 84(44.90), \text { VNR } 28 \times M C \\
84(39.47), \text { PDM } \times \text { VNR } 22(2.25)\end{array}$ & $\begin{array}{l}\text { VNR 28× MC84 (143.04), PDM } \times \text { VNR } 22 \\
\text { (113.62), VNR } 28 \times \text { Pant Karela } 3(98.15)\end{array}$ & $\begin{array}{l}\text { VNR } 22 \times \text { MC } 84(85.29), \text { MC } 84 \times \text { Pant Karela } \\
3(81.09), \text { VNR } 28 \times \text { MC } 84(42.96)\end{array}$ \\
\hline 12. & Fruit Length $(\mathrm{cm})$ & $\begin{array}{l}\text { MC } 84 \times \text { PBIG 2(5.17), VNR 22× US 33(3.71), } \\
\text { PDM ×PBIG } 3 \text { (2.39) }\end{array}$ & - & $\begin{array}{l}\text { MC84× PBIG } 2 \text { (27.68), PDM ×US } 33 \text { (14.70), } \\
\text { PDM ×PBIG } 2 \text { (14.06) }\end{array}$ \\
\hline 13. & Fruit Dia $(\mathrm{cm})$ & $\begin{array}{l}\text { VNR } 28 \times \text { PBIG } 2(0.72), \text { VNR } 22 \times \text { Pant } \\
\text { K1 }(0.45), \text { PDM } \times \text { US } 33(0.44)\end{array}$ & $\begin{array}{l}\text { VNR } 28 \times \text { PBIG } 2 \text { (22.94), PDM } \times \text { US33 (10.40), } \\
\text { PDM } \times \text { MC } 84(8.99)\end{array}$ & $\begin{array}{l}\text { VNR } 22 \times \text { Pant Karela } 1 \text { (14.79), VNR } 28 \times \text { PBIG } 2 \\
\text { (9.47), PDM } \times \text { Pant Karela } 3 \text { (7.80) }\end{array}$ \\
\hline 14. & Length/ Dia ratio & $\begin{array}{l}\text { Pant Karela } 3 \times \text { PBIG 2(10.01), MC } 84 \times \text { Pant K } \\
3(11.03)\end{array}$ & - & MC 84×PBIG 2 (36.89) \\
\hline 15. & Average Fruit Weight & $\begin{array}{l}\text { Pant Karela } 3 \times \text { PBIG 2(10.80), MC84× Pant } \\
\text { Karela 3(13.51), PDM } \times \text { VNR } 28(11.14)\end{array}$ & $\begin{array}{l}\text { PDM×MC84 (5.63),, MC 84×Pant Karela } 3 \text { (3.99), } \\
\text { Pant Karela } 1 \times \text { US } 33(2.93)\end{array}$ & $\begin{array}{l}\text { US } 33 \times \text { PBIG } 2 \text { (11.62),, VNR 22× US } 33 \text { (5.73), } \\
\text { MC } 84 \times \text { Pant Karela } 3(3.99)\end{array}$ \\
\hline 16. & Fruits/ Plant & $\begin{array}{l}\text { VNR 28×Pant Karela 3(21.66), VNR 28× MC } \\
\text { 84(10.45), VNR 22×MC 84(19.78) }\end{array}$ & $\begin{array}{l}\text { VNR 28×Pant Karela 3(160.55), VNR 22×MC } 84 \\
\text { (130.26), VNR 28×MC } 84 \text { (127.27) }\end{array}$ & $\begin{array}{l}\text { Pant Karela } 1 \times \text { Pant Karela } 3 \text { (57.42), VNR } 22 \times \text { MC } \\
84(50.02) \text {, VNR } 28 \times \text { Pant Karela } 3(29.02) .\end{array}$ \\
\hline 17. & Fruit Yield//Plant (gm) & $\begin{array}{l}\text { US } 33 \times \text { Pant Karela } 3 \text { (346.95), VNR22× } \\
\text { PantKarela1(398.51), MC } 84 \times \text { Pant Karela } 3 \\
\text { (264.74) }\end{array}$ & $\begin{array}{l}\text { US33×Pant Karela } 3 \text { (29.34), MC84× US } 33 \\
(21.97), \text { PDM } \times \text { US } 33(21.17)\end{array}$ & $\begin{array}{l}\text { US } 33 \times \text { Pant Karela } 3 \text { (29.34), VNR } 22 \times \text { US33 } \\
\text { (15.32), US } 33 \times \text { PBIG } 2 \text { (11.22) }\end{array}$ \\
\hline 18. & Fruit Yield/ hac & $\begin{array}{l}\text { US } 33 \times \text { Pant Karela } 3(14.47) \text { VNR } 22 \times \text { Pant } \\
\text { Karela } 1(17.07), \text { MC } 84 \times \text { Pant Karela } 3(11.05)\end{array}$ & $\begin{array}{l}\text { US } 33 \times \text { Pant Karela } 3(29.34), \text { MC } 84 \times \text { US } 33 \\
(21.97), \text { PDM } \times \text { US 33(21.18) }\end{array}$ & $\begin{array}{l}\text { US33×Pant Karela } 3 \text { (29.34), VNR } 22 \times \text { US } \\
\text { 33(15.32), US 33× PBIG } 2 \text { (11.22) }\end{array}$ \\
\hline
\end{tabular}


The significance of SCA effects elucidates the presence of genetic diversity among parents tested and illustrates the contribution of dominance/ epistatic effect which represents the non fixable components of genetic variation related to heterosis. The crosses showing sca effects involving parent with good gca could be exploited as $F_{1}$ hybrid breeding, however if a cross having high sca has one of its parents as good general combiner and the other as poor or average combiner, such crosses are likely to give some segregants. These results were in the conformity with the results reported by Masmade and Kale (1986) who evaluted combining ability in seven cultivars of cucumber crossed in diallel fashion excluding reciprocals and found that both GCA and SCA variances were significant for all the characters. The hybrids Poona Khira X Japanese Long Green, White Long Cucumber X Poinsette, Kalyanpur Ageti X Panval and Poona Khira X Turkish Long Green were found to be most promising as having highest SCA effects. Jankiram and Sirohi (1988) also estimated components of SCA in bottle gourd hybrids. The F1 hybrid S-46 X S-54 was the best specific combiner for fruit weight and total yield per plant. Vahab (1989) observed highest SCA effect for total yield and number of fruits per vine in cross, Arka Harit $\times$ MAC79.

\section{Heterosis}

There is a good scope of exploiting heterosis in bitter gourd because of the fact that it is a cross pollinated crop. In the present study, the extent of heterosis was studied in $28 \mathrm{~F} 1$ hybrids of bitter gourd developed by 8 parents in diallel design in two seasons. The estimates of heterobeltiosis (better parent) and standard heterosis (check parent) have been presented in table 3. For the characters days to $1^{\text {st }}$ female flower anthesis and node number to $1^{\text {st }}$ female flower, the negative heterosis was considered to be desirable, as it indicates earliness. The parental genotype PBIG 4 (Pant Karela 3) was used as a check for standard heterosis. For days to first female flower, the heterobeltiosis raged from -16.82 (MC84×PBIG2) to -4.18 (VNR22× US33). The highest negative values was obtained in $\begin{array}{llll}\text { crosses } & -16.82 \quad \text { (MC84×PBIG2), } & -14.74\end{array}$

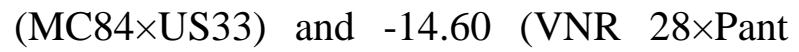
karela 3). Standard heterosis was found maximum in MC $84 \times$ PBIG2 (-21.46), $\mathrm{PDM} \times \mathrm{MC} 84(-19.74)$ and PDM $\times \mathrm{VNR} 28(-$ 19.32) in season I. In season II, maximum negative heterobeltiosis, was found in crosses VNR28× MC84 (-24.93), VNR28× US33 (24.25) and PDM $\times$ Pant karela 3 (-20.63). For heterosis over check parent, top crosses were VNR28× US33 (-28.94), VNR28× MC84 (28.72) and PDM $\times$ VNR28 (-27.97). Pooled data revealed that crosses VNR 28x US33 (20.00), VNR28 $\times$ Pant karela $3(-15.60)$ and VNR $28 \times$ MC84 (-13.19) had maximum value for heterobeltiosis. Over check parent the crosses PDM $\times$ VNR28 (-23.95), VNR $28 \times$ MC $84(-21.95)$ and VNR 28× US33 (19.77) showed heterotic effects. The magnitude of heterobeltiosis for node number to first female flower ranged from -47.86 to 0.02 , out of which the top three crosses were VNR28 $\times$ VNR22 (-47.86), MC84 × US $33(-$ 40.96) and VNR $28 \times$ Pant karela 1 (-34.75). Over the standard check (Pant karela 3), top three crosses havng maximum heterosis were VNR $28 \times$ VNR 22 (-40.20), VNR 28×US33 (36.76) and VNR 28× MC84 (-32.35) in 2014. For year 2015, maximum value for heterobeltiosis was noted for crosses VNR28×US33 (-50.28), MC $84 \times$ US33 (39.26) and VNR28×Pant karela 3 (-33.52). The magnitude of standard heterosis was found maximum for MC 84×PBIG2 (-44.87), VNR $28 \times$ MC84 (-40.37) and VNR $28 \times$ US33 (-36.30). For pooled season, maximum heterosis over better parent was found in crosses VNR28 $\times$ US33 (-55.87), MC84×US $33(-40.13)$ and VNR $28 \times$ VNR 22 (- 26.08). 
Standard heterosis was found maximum in crosses VNR 28× MC 84 (-36.72), VNR 28× US $33(-36.51)$ and VNR 28xPBIG 2 (29.44).

Out of all the 28 crosses, three crosses showed significant positive heterobeltiosis in season I viz., US 33×PBIG 2 (12.04), VNR $22 \times$ US 33 (5.25) and MC 84× Pant karela 3 (5.02) for fruit weight. Crosses which found to have significantly positive heterosis over standard check were US 33×PBIG 2 (9.17), VNR $22 \times$ US 33 and US $33 \times$ Pant karela 3 (2.56) and Pant karela 1× US 33 (2.14). For season II, US $33 \times$ PBIG 2 (11.18), Pant karela $1 \times$ US 33 (9.19) and VNR $22 \times$ Pant karela 1 (6.22) showed significant positive values for heterobeltiosis. Whereas for standard heterosis very little amount was noticed in crosses PDM $\times$ MC84 (9.45), Pant karela 1× US33 (3.66) and PDM× PBIG 2 (1.92) respectively. Pooled data revealed comparatively less than $10 \%$ of heterosis over better parent and standard check viz., US33x PBIG 2 (11.62), VNR 22× US 33 (5.73), MC $84 \times$ Pant karela 3 (3.99) and PDM $\times$ MC84 (5.63), MC $84 \times$ Pant karela 3 (3.99), Pant karela $1 \times$ US 33 (2.930), respectively. For number of fruits per plant, a significant amount of heterobeltiosis was observed among crosses VNR $28 \times$ Pant karela 3 (68.26), Pant karela $1 \times$ Pant karela 3 (54.31) and VNR $28 \times$ MC 84 (48.82) in season I. Crosses exhibiting highest amount over standard heterosis were VNR 28× Pant karela 3 (204.88), VNR 28× MC 84 (169.65) and VNR 28× Pant karela 1 (140.71). For next season, magnitude of heterobeltiosis was found maximum for Pant karela $1 \times$ Pant karela 3 (60.54), VNR 22× MC 84 (53.12) and PDM $\times$ Pant karela 1 (19.64). For standard heterosis, highest significant positive values were obtained for crosses VNR $22 \times$ MC 84 (139.44), VNR 28×P ant Karela 3 (112.64) and VNR 28× MC 84 (81.46). On pooling data it was observed that the top three crosses which were having significant positive amount of heterosis over better parent were Pant karela 1× Pant karela 3 (57.42), VNR $22 \times$ MC 84 (50.02) and VNR 28× Pant karela 3 (29.02). For heterosis over standard check, crosses found were VNR $28 \times$ Pant karela 3 (160.55), VNR $22 \times$ MC 84 (130.26) and VNR $28 \times$ MC 84 (127.27).

In 2014, the crosses which exhibited significant high magnitude of heterobeltiosis were US33 $\times$ Pant karela 3 (32.29), US33×PBIG 2 (24.01) and VNR 22× US33 (18.65). Values for standard heterosis were found maximum for the crosses US33 $\times$ Pant karela 3 (32.29), PDM× US 33 (31.60) and VNR 22x US33 (26.67). In 2015, the values for heterosis over better parent were found highest in crosses US33 $\times$ Pant karela 3 (26.40), VNR 22×Pant karela 1 (13.98) and VNR 22× US33 (11.82), respectively. For standard heterosis, crosses US33 $\times$ Pant karela 3 (26.40), MC $84 \times$ US33 (24.41) and MC $84 \times$ Pant karela 3 (18.49) revealeded highest values. Pooled data revealed that US33 $\times$ Pant karela 3 (29.34), VNR 22× US 33 (15.32) and US 33× PBIG 2 (11.22) exhibited significant amount of heterobeltiosis. Maximum standard heterosis for fruit yield was found highly significant and positive for the crosses US 33× Pant karela 3 (29.34), MC $84 \times$ US 33 (21.97) and PDM $\times$ US 33 (21.18).

The yields in F1 hybrids have been attributed to earliness, increased no. of fruits per plant and increase in fruit weight. The results of present investigation are similar to the findings of Tewari and Ram (1999) in studying heterosis for yield and other associated characters in bitter gourd using three $F_{1}$ hybrids from 3 promising genotypes (PBIG-1, PBIG-2 and PBIG-3) of diverse nature reported ample amount of heterosis for yield over local check and better parent. The best performing hybrid was PBIG-1 $\times$ PBIG-2 which showed 25.75 per cent heterosis over 
better parent. Rajeswari and Natarajan (1999) evaluated 30 hybrids of bitter gourd in full diallel fashion and reported significant heterosis for sex ratio, fruit length, fruit girth, fruit weight, number of fruits per hill, yield per hill. The Hybrids, Preethi $\times$ MDU-1, Preethi $\times$ Co. 1 and Arka Harit $\times$ Preethi had highest heterosis for yield per hill and fruit weight. Singh et al., (2000) also evaluated seven parental lines (BG-4, BG-11, BG-23, BG-25; Pusa Do-Mausami, BG-29, BG-46 and BG-52) and their $21 \mathrm{~F}_{1}$ hybrids of bitter gourd in half diallel fashion and they observed that BG-5, BG-23 and BG-11 were the three top performing parents for fruit yield per plant. Tamilselvi et al., (2015) conducted an experiment to study the heterosis for earliness and yield characters. Evaluation of parents revealed that the parents Kashi Harit, Vadhalagundu Local, and $\mathrm{CO}_{2}$ were identified as the best genotypes for improvement of yield and earliness

\section{References}

Suthar, S., 2007. Vermicomposting potential of Perionyx sansibaricus (Perrier) in different waste materials. Bioresour. Technol. 98, 1231-1237. Prakash, M., and Karmegam, N.2010.Vermistabilization of pressmud using Perionyx ceylanensis Mich. Bioresource Technology. 101 (21): 8464-8468.

Vahab, A. 1989. Homeostatic analysis of components of genetic variance and inheritance of fruit colour, fruit shape and bitterness in bitter gourd. Ph. D. Thesis. Kerala Agril. Univ., Vellanikkara, Trichur

Devadas, V.S. 1993. Genetic studies on fruit and seed yield and quality in bitter gourd (Momordica charantia L.). Ph.D. (Hort.) thesis, TNAU, Coimbatore
Dey, S.S., Behera, T.K., Munshi, A.D. and Pal, Anand. 2010. Combining ability analysis in bitter gourd. Indian J. Hort., 67 (3): 399-401.

Gilbert, N. 1967. Additive combining abilities fitted to plant breeding data. Biometrics. 23:45-50.

Griffing B. 1956. Concept of general and specific combining ability in relation to diallel crossing systems. Australian Journal of Biological Sciences. 9:463493.

Gopalakrishnan, R. 1986. Diallel analysis in bitter gourd (Momordica charantia L.), M.Sc. (Hort.) thesis, TNAU, Coimbatore, India

Gupta, V., Kishore, N. and Partap, P.S. 2006. Diallel analyses for combining ability in bitter gourd (Momordica charantia Linn.). Crop Res. 32: 406-410.

Janakiram, T. and Sirohi, P.S. (1989). Heterosis studies in long fruited bottle gourd. Madras Agric. J. 76: 339-342.

Bhatt, L., Singh, S.P., Soni, K. and Samota, M.K. 2017. Combining Ability Studies in Bitter Gourd (Momordica charantia L.) for Quantitative Characters. International Journal of Current Microbiology and Applied Sciences. Vol 6(7): 4471-4478

Masmade, A.M. and Kale, P.N. 1986. Heterosis and combining ability in cucumber (Cucumis sativus L.). Veg. Sci. 13.1:60-68

Rajeswari, K.S. and Natarajan, S. 1999. Studies on heterosis for growth, yield and quality parameters in bitter gourd (Momordica charantia L.). South Indian J. Hort., 47(1-6): 208-209.

Schaefer and Renner. 2010. A three-genome phylogeny of Momordica (Cucurbitaceae) suggests seven returns from dioecy to monoecy and recent long-distance dispersal to Asia. 54(2):553-60. 
Singh, K.P., Choudhary, D.N., Singh, V.K. and Mangal, G. 1995. Studies on combining ability in bottle gourd. Veg. Sci. 22: 101-104.

Singh, P. and Narayanan, S.S. 1993. Biometrical Techniques in Plant Breeding. Kalyani publishers, New Delhi. Pp. 187.

Srivastava, V. and Nath, P. 1983. Studies on heterosis in bittergourd (Momordica charantia L.). Egyptian journal of genetics and cytology 12(2): 317-327.

Snedecor, G. W. and Cochran, W. G. 1967. Statistical methods applied to experiments in agriculture and biology. 5th ed. Ames, Iowa: Iowa State University Pres

Tamilselvi, N.A., Jnsirani, P. and Pugalendhi, L. 2015. Estimation of heterosis and combning ability for earliness and yieldcharacters in pumpkin. Vol.10(16).pp. 1904-1912.

Tewari, D. and Ram, H.H. 1999. Heterosis in bitter gourd (Momordica charantia L.). Veg. Sci., 26(1): 27-29.

\section{How to cite this article:}

Vibha Mishra and Singh, D.K. 2018. Combining Ability and Heterosis Studies in Bitter Guard (Momordica charactia L.). Int.J.Curr.Microbiol.App.Sci. 7(07): 4278-4289. doi: https://doi.org/10.20546/ijcmas.2018.707.499 